\title{
Evaluación del Proceso de Convergencia Europea desde la Perspectiva de Estudiantes del Máster de Secundaria
}

\author{
European Convergence Process Evaluation from the Perspective \\ of Master`s Degree Programme for Secondary Education
} Students

\author{
$\mathrm{M}^{\mathrm{a}}$ del Carmen Pegalajar Palomino* \\ Universidad de Jaén
}

\begin{abstract}
El proceso de convergencia europea en el ámbito de la Educación Superior ha supuesto una transformación de la estructura organizativa. De este modo, la formación inicial del docente para la etapa de Educación Secundaria se ha organizado en torno al desarrollo de un máster. En este contexto, el punto de vista del estudiante, sus percepciones, expectativas y necesidades suponen un indicador de evaluación de calidad del proceso educativo. Por ello, esta investigación pretende conocer las percepciones de los estudiantes del Máster en Formación del Profesorado en Educación Secundaria Obligatoria de la Universidad Católica de Murcia acerca del proceso de convergencia europea. Para ello, se ha utilizado una metodología descriptiva, llevándose a cabo la recogida de datos mediante un cuestionario ad hoc $(\mathrm{n}=252)$. El alumnado valora favorablemente el proceso de cambio hacia el sistema europeo en Educación Superior. Asimismo, se destaca la existencia de diferencias estadísticamente significativas en sus valoraciones, siendo estas más favorables en estudiantes de más edad así como en aquellos que cursan dicho título por la especialidad de Filología e Idiomas y Humanidades, Ciencias Sociales y de la Empresa.
\end{abstract}

Palabras clave: Máster, Alumno, Sistema de enseñanza, Enseñanza postobligatoria, Reforma educativa, Evaluación.

\begin{abstract}
European convergence process in higher education has involved an organizational structure transformation. Thus, the initial training of teachers for secondary education stage is organized around the development of a master degree. In this context, the point of view of the student, their perceptions, expectations and needs are an indicator of educational quality assessment process. Thus, this research analyzes perceptions about European convergence process of students of master`s degree programme for secondary education from the Catholic University of Murcia. It is used a descriptive methodology, carrying out data collection through an ad hoc questionnaire $(\mathrm{n}=252)$. Students positively assessed the change process towards the European Higher Education system. Similarly, the existence of statistically significant differences in their rating stands, these being more favorable in older students and those who attend this degree in the specialty of Philology and Languages and Humanities, Social Sciences and Business.
\end{abstract}

Keywords: Master, Student, Education system, Post-compulsory education, Educational reform, Evaluation. 


\section{Introducción}

La instauración del proceso de convergencia europea en el ámbito de la Educación Superior en España ha supuesto la redefinición y transformación de las estructuras organizativas en un modelo de enseñanza-aprendizaje más flexible, dinámico, creativo e innovador (De Pablos, Colás, González y Jiménez, 2007) pero, a la vez, coherente y sensible a las demandas sociales y necesidades formativas del alumnado (González, 2008). Dicha transformación se concreta, tal y como establecen Gutiérrez-Herrera et al. (2012), en el diseño de un nuevo contexto educativo, social, cultural y tecnológico en que se desenvuelven las instituciones universitarias, el cual ha contribuido a establecer nuevas exigencias en la formación de los universitarios, quienes no solo deben aprender contenidos curriculares propios de su formación, sino nuevas competencias profesionales que permitan ajustarse de forma dinámica a la transformación del escenario social.

La Educación Superior se sitúa, por tanto, ante una concepción del aprendizaje constructivista, basada en el aprendizaje significativo y la autonomía del estudiante, capaz de conocer sus propios procesos cognitivos y controlar su aprendizaje (Olmedo, 2013). De esta forma, enseñanza y aprendizaje se funden mutuamente en una completa y estrecha dinámica, en la que las interacciones se suceden de manera multilateral e indisoluble para mejorar la calidad del proceso educativo (López, Pérez-García y Rodríguez, 2015). Ante esta nueva situación, tal y como afirman Fernández-Molina, González y Del Molino (2011), la nueva metodología y trabajo por competencias que supone el Espacio Europeo de Educación Superior exige al alumno nuevos roles y responsabilidades, llevándole a perfeccionar o modificar competencias, creencias o ideas sobre la Educación Superior y los estudios que realiza. Asimismo, Hargreaves y Shirley (2012) exponen cómo el éxito de las reformas en educación requiere de un mayor compromiso por parte de los agentes implicados, entre los que se encuentran alumnado, profesorado, gestores, etc.

Los cambios estructurales y metodológicos vinculados a la implantación del Espacio Europeo de Educación Superior en la universidad española han convertido la formación docente en un ámbito relevante en las universidades (Perales, Jornet y González-Such, 2014). Así pues, la formación inicial del docente para la etapa de Educación Secundaria Obligatoria se ha organizado en torno al desarrollo de un Máster Universitario en Profesorado de Educación Secundaria Obligatoria y Bachillerato, Formación Profesional y Enseñanza de Idiomas. Dicha titulación, más alineada con las prácticas y tendencias de los países de la Unión Europea (Cid, Sarmiento y Pérez, 2012), ha permitido acometer la necesaria renovación del modelo de formación inicial docente (Medina, 2010), paliando algunas de las deficiencias estructurales, organizativas e incluso conceptuales que han venido arrastrando el sistema de formación inicial del profesorado de Secundaria desde 1970 con la implantación del Curso de Aptitud Pedagógica (Imbernón, 2007).

Dichos estudios suponen una oportunidad formativa para futuros docentes (González Sanmamed, 2009) centrada en el desarrollo de competencias y conocimientos psicopedagógicos y didácticos más específicos (González, Jiménez y Pérez, 2011); se ha planteado como una alternativa para la contribución a la renovación de la enseñanza (Vilches y Gil-Pérez, 2010). En este proceso, el futuro docente debe conocer los aspectos sociales de la enseñanza a través del apoyo a la formación de la identidad docente (Timostsuk y Ugaste, 2010). 
Así pues, supone un reto la mejora de la formación de los docentes para la etapa de Educación Secundaria (Puelles, 2009), hasta el momento escasamente formados en materias pedagógicas, organizativas e institucionales propias de la función docente (Benso y Pereira, 2003). Este hecho ha provocado un aumento del interés de los investigadores sobre la temática (Manso-Ayuso y Martín, 2014; Pérez y Rodríguez, 2012; Sans de Castro, 2013), tratando de analizar las percepciones del alumnado del Máster de Secundaria acerca del proceso de convergencia europea.

La visión del estudiante, producto de sus percepciones, expectativas y necesidades, sirve como indicador de evaluación de la calidad y, en definitiva, de la mejora en la gestión y el desarrollo de la Educación Superior (Álvarez, Chaparro y Reyes, 2015). De igual modo, Lukas, Santiago, Etxeberria y Lizasoain (2014) definen cómo las valoraciones del alumnado sobre la docencia de su profesorado y sus actuaciones son un indicador de la calidad docente. Desde esta perspectiva, la evaluación debe entenderse como un proceso de intercambio y comprensión de información sobre los acontecimientos que tienen lugar en el aula universitaria, con la finalidad de crear estrategias docentes que mejoren tanto el aprendizaje del alumnado como la formación docente (Alcaraz, Fernández y Sola, 2012).

Así pues, autores como Castaño, Benito, Portela y Rodríguez (2007) y Otero, Ferro y Vila (2012) subrayan cómo la transformación sufrida en el ámbito de la Educación Superior ha mejorado la satisfacción del alumnado respecto al profesorado y la aplicación de conocimientos. Entre las cuestiones más positivamente valoradas destacan la evaluación continua, la relación personalizada con el docente, la posibilidad de estudiar o trabajar en otros países europeos y la disminución de la ratio, a pesar de criticar la obligatoriedad de la asistencia a clase y el exceso de trabajos individuales o en grupo (Carrasco, 2012). En esta misma línea, Serrano y Pontes (2015) destacan cómo la puesta en marcha del máster ha generado buenas expectativas sobre el nuevo modelo formativo, vinculadas al fomento de valores sociales, culturales y educativos en el alumnado.

No obstante, otras investigaciones (Font-Mayolas y Masferrer, 2010; Leo y Cubo, 2012; Méndez, 2008) revelan la escasa información de que dispone el alumnado acerca de la instauración del Espacio Europeo de Educación Superior, repercutiendo de forma negativa en el rol que estos pueden adoptar dentro del proceso de convergencia europea. Así pues, autores como Coterón, Franco y Gil (2012) han demostrado la valoración negativa del alumnado hacia los cambios introducidos por el nuevo modelo europeo en Educación Superior. En este sentido, Cachón, López, Romero, Zagalaz y González (2015) ponen de manifiesto diferencias entre profesores y alumnos al cuestionar la labor docente y las expectativas de aquellos que se encuentran desarrollando su período formativo.

De igual modo, Benarroch (2011) describe las valoraciones de expertos en la formación de profesores de Educación Secundaria acerca del máster, destacando como principales debilidades la ausencia de criterios de temporalización entre módulos así como la falta de coherencia entre los modelos de enseñanza utilizados y los que se pretenden que los futuros profesores apliquen en sus aulas. No obstante, los propios alumnos perciben la importancia de que el profesor de Educación Secundaria esté formado tanto en aspectos curriculares como afectivos y habilidades relacionales (Reoyo, Carbonero, Freitas y Valdivieso, 2012). Además, el alumnado del Máster de Secundaria no se muestra satisfecho con el tiempo establecido para su formación didáctica, la vinculación entre 
teoría y práctica en el desarrollo de programas formativos así como con la coordinación entre el profesorado, criticando el desarrollo de prácticas docentes demasiado individualistas (Hernández y Carrasco, 2012).

Tomando como referencia los estudios e investigaciones analizados, se plantea conocer las percepciones de futuros docentes de Educación Secundaria acerca de este nuevo proceso en Educación Superior. Sus evaluaciones deben servir a los profesionales de la educación para conocer las potencialidades y limitaciones del sistema y, de este modo, mejorar la calidad de la educación universitaria en el contexto europeo. Dicho concepto aparece ligado a la idea de excelencia, perfección, adecuación a un propósito preestablecido o transformación del sistema tal y como establecen Rué, Mateo y Muñoz (2012).

\section{Método}

Esta investigación pretende conocer las percepciones del alumnado del Máster de Secundaria de la Universidad Católica de Murcia acerca del proceso de convergencia europea. De igual modo, se pretende analizar la existencia o no de diferencias estadísticamente significativas en dichas valoraciones según la edad del alumnado que cursa dicho título así como la especialidad desde la que realizan el Máster en Formación del Profesorado en Educación Secundaria Obligatoria y Bachillerato, Formación Profesional y Enseñanza de Idiomas. Para ello, se ha utilizado una metodología de tipo descriptivo, llevándose a cabo la recogida de datos mediante la técnica de la encuesta.

\subsection{Descripción de la muestra}

La población objeto de estudio está compuesta por 264 estudiantes matriculados durante el curso 2013-2014 en el Máster de Profesorado en Educación Secundaria Obligatoria y Bachillerato, Formación Profesional y Enseñanza de Idiomas de la Universidad Católica de Murcia. La selección de los sujetos se ha realizado a partir de un muestreo probabilístico aleatorio simple, de forma que han participado todos aquellos que al azar respondieron a los cuestionarios enviados $(\mathrm{n}=252)$. Para el cálculo de la muestra participante se ha utilizado la fórmula para poblaciones con menos de 100.000 sujetos con un nivel de confianza del 95\% y un error de estimación máximo de un $4 \%$. Se trata, por tanto, de una muestra con un tamaño pequeño asociada a una universidad pública, lo que limita la generalización de las conclusiones aportadas en el estudio para otras muestras. En la tabla 1 se presentan algunas de sus características más destacadas.

Tabla 1. Características de la muestra

\begin{tabular}{ll}
\hline \multirow{2}{*}{ GÉNERO } & Mujeres: $59,1 \%$ \\
& Hombres: $40,4 \%$ \\
\hline \multirow{3}{*}{ EDAD } & $20-25$ años: $43,3 \%$ \\
& $26-30$ años: $33,7 \%$ \\
& Más de 30 años: $23,0 \%$ \\
\hline \multirow{2}{*}{ ESPECIALIDAD } & Humanidades, Ciencias Sociales y Empresa: 32,7\% \\
& Ciencia y Tecnología: $27,5 \%$ \\
& Actividad Física y del Deporte: $20,3 \%$ \\
& Filología e Idiomas: $17,5 \%$ \\
& Artes: $2,0 \%$ \\
\hline \multirow{2}{*}{ Motivo ACCESO AL MÁsTER } & Ser profesor de Educación Secundaria: $75,7 \%$ \\
& Acceder a estudios de Doctorado: $14,9 \%$ \\
& Conseguir formación psicopedagógica complementaria: $9,5 \%$ \\
\hline
\end{tabular}

Fuente: Elaboración propia. 


\subsection{Instrumento de recogida de datos}

La recogida de datos se ha realizado a través del "Cuestionario sobre las actitudes del alumnado universitario hacia el Espacio Europeo de Educación Superior”. Se trata de una escala tipo Likert cuyas respuestas oscilan entre 1 y 5 (siendo $1=$ totalmente en desacuerdo y $5=$ totalmente de acuerdo), compuesto por seis dimensiones fundamentales: reestructuración académica en el Espacio Europeo de Educación Superior; planificación docente; convergencia europea en los planes de estudio; organización docente; información académica y especialización de los títulos. Además, el cuestionario cuenta con un bloque inicial de información identificativa del alumnado con el que se pretende conocer datos como el sexo, la edad, la especialidad y el motivo por el que cursa dicho título.

Una vez la escala se ha construido, se ha realizado el análisis de la fiabilidad y validez del instrumento. Así pues, y para comprobar la validez de contenido, se ha contado con una docena de expertos universitarios en el área de Didáctica y Organización Escolar (DOE) de la Universidad de Jaén y Granada, a los que se les pidió que valoraran y validaran la pertinencia de cada ítem del cuestionario, estableciendo las consideraciones oportunas para su mejora en cuanto a la pertinencia de los ítems, ambigüedad, redacción y otros aspectos de interés.

Una vez las sugerencias de los expertos se incorporaron en la escala, se aplicó la escala a una muestra piloto conformada por cincuenta estudiantes de tercer curso del Grado de Educación Infantil de la Universidad de Jaén, los cuales no han sido incorporados en la muestra final de este estudio. Dicha prueba pretende conocer el grado de comprensión de los distintos ítems por parte del alumnado y analizar la validez de constructo. Así, el índice de adecuación muestral KMO alcanza un valor de 0.72 y la prueba de esfericidad de Bartlett es de 2149.940 ( $\mathrm{p}=.000$ ). Estos datos hacen que se rechace la hipótesis nula de que la matriz de correlación inter-ítems es identidad y se considera que las respuestas están sustancialmente relacionadas. El análisis de los componentes principales revela, tras la rotación Varimax, la convergencia de seis factores que explican el 52,44\% de la varianza; los ítems muestran valores apropiados, situándose entre .625 y .838. Dichos factores coinciden con las seis dimensiones del cuestionario anteriormente descritas y que pretenden analizar las percepciones del alumnado universitario acerca del proceso de convergencia europea en Educación Superior.

Por su parte, el estudio de la fiabilidad confirma la alta consistencia interna del instrumento al alcanzar el coeficiente Alfa de Cronbach el valor de .934, lo que indica que la escala diseñada es muy confiable. Además, para el método de las dos mitades se obtiene para la primera parte un valor de .884 y para la segunda un valor de .910 , lo que vuelve a corroborar los resultados obtenidos en el procedimiento anterior.

\section{Resultados}

\subsection{Análisis descriptivo}

A nivel general, y tras el análisis de la media global del cuestionario, se aprecian resultados positivos por parte del alumnado encuestado $(\mathrm{M}=3,52 ; \mathrm{SD}=, 954)$. La muestra valora de manera favorable el proceso de cambio hacia el sistema europeo en Educación Superior. Además, y al comparar las medias para cada dimensión (tabla 2), se considera cómo los mejores resultados corresponden con las valoraciones del alumnado hacia las 
posibilidades de especialización de que disponen para el título que están cursando $(\mathrm{M}=3,84 ; \mathrm{SD}=, 900)$, así como sus percepciones hacia la planificación docente llevada a cabo en dicho máster $(\mathrm{M}=3,64 ; \mathrm{SD}=, 962)$ y la nueva reestructuración académica de Educación Superior desarrolladas gracias al proceso de convergencia europea $(\mathrm{M}=3,61$; $\mathrm{SD}=0,954)$. También se han obtenido resultados favorables para las percepciones del alumnado hacia la planificación y mejora del proceso de enseñanza-aprendizaje $(\mathrm{M}=3,55$; $\mathrm{SD}=1,142)$, información académica acerca del máster que cursan $(\mathrm{M}=3,37 ; \mathrm{SD}=, 851)$ y conocimiento acerca del proceso de convergencia europea en dicho plan de estudios $(\mathrm{M}=3,30 ; \mathrm{SD}=, 845)$.

Tabla 2. Análisis de medias globales para cada dimensión

\begin{tabular}{lcc}
\hline & M & SD \\
\hline Reestructuración académica en el EEES & 3,61 &, 954 \\
Planificación docente & 3,64 &, 962 \\
Convergencia europea en los planes de estudio & 3,30 &, 845 \\
Organización docente & 3,55 & 1,142 \\
Información académica & 3,37 &, 851 \\
Especialización de los títulos & 3,84 &, 900 \\
\hline
\end{tabular}

Fuente: Elaboración propia.

Para el factor "Reestructuración académica en el EEES" (tabla 3), los resultados obtenidos por el alumnado son bastante favorables. El único aspecto en el que los estudiantes del Máster de Secundaria se muestran menos convencidos es el hecho de que la nueva reestructuración de las titulaciones en grado y máster haya supuesto una mejora de la educación universitaria $(\mathrm{M}=2,96 ; \mathrm{SD}=1,170)$. Así pues, y desde la perspectiva del alumnado, se cuestiona la repercusión del proceso de convergencia europea en la enseñanza universitaria, lo cual ha supuesto la modificación de la actual estructura del sistema en Educación Superior. Una de las principales repercusiones ha sido la modificación flexible de los grados (con cuatro años de duración) y los másteres (con una duración de un año), los cuales tienden a converger con la mayoría de los países europeos.

No obstante, consideran que la distribución del plan de estudios del Máster de Secundaria les permite desarrollar un proceso de enseñanza de calidad ( $M=3,50$; $\mathrm{SD}=0,913)$, valorándolo de manera positiva $(\mathrm{M}=3,61 ; \mathrm{SD}=942)$. Del mismo modo, avalan la importante labor desempeñada por la facultad $(\mathrm{M}=3,65 ; \mathrm{SD}=, 934)$ y la propia universidad $(\mathrm{M}=3,83 ; \mathrm{SD}=, 877)$ así como la calidad de la enseñanza del máster que cursan $(\mathrm{M}=3,69 ; \mathrm{SD}=, 926)$. Al tratarse de estudiantes de posgrado, su experiencia previa con otros estudios universitarios permite dar a conocer determinados indicadores prácticos que definan el concepto de mejora educativa en el sistema universitario, desde el prisma de la calidad e innovación docente.

En cuanto al profesorado, los estudiantes consideran que este cumple con sus expectativas formativas ( $\mathrm{M}=3,74 ; \quad \mathrm{SD}=, 924)$ y necesidades educativas $(\mathrm{M}=3,80$; $\mathrm{SD}=, 946)$, siendo las guías docentes las que permiten un mayor conocimiento de la asignaturas $(\mathrm{M}=3,77 ; \mathrm{SD}=, 860)$. Estas suponen un manual fundamental de la asignatura elaborado por el docente y que contiene información acerca de los objetivos, contenidos, tareas y actividades, bibliografía, temporalización, etc., lo que facilita el proceso de aprendizaje del alumnado, poniendo a su disposición información de calidad para su mejor desempeño académico. A su vez, el docente permite dar a conocer aspectos 
fundamentales de la enseñanza que facilitan la planificación, organización y reflexión previa al desarrollo del proceso.

Tabla 3. Análisis descriptivo del factor "Reestructuración académica en el EEES"

\begin{tabular}{lcc}
\hline \multicolumn{1}{c}{ ÍTEMS } & M & SD \\
\hline $\begin{array}{l}\text { La reestructuración de las titulaciones en Grado y Máster mejora la calidad } \\
\text { de la educación universitaria }\end{array}$ & 2,96 & 1,170 \\
$\begin{array}{l}\text { La distribución del plan de estudios del máster permite desarrollar un } \\
\text { proceso de enseñanza de calidad }\end{array}$ & 3,50 &, 913 \\
Mi valoración acerca del plan de estudios del máster es positiva & 3,61 &, 942 \\
La facultad desempeña una labor importante en el plan de estudios del & 3,65 &, 934 \\
máster & 3,69 &, 926 \\
La enseñanza recibida en el máster es de calidad & 3,74 &, 924 \\
$\begin{array}{l}\text { En líneas generales, el profesorado del título cumple con mis expectativas } \\
\text { formativas }\end{array}$ & 3,77 &, 860 \\
Las guías docentes me permiten un mayor conocimiento de las asignaturas & 3,80 &, 946 \\
Percibo que el profesorado del título da respuesta a las necesidades & & \\
educativas del alumnado & 3,83 &, 877 \\
En líneas generales, mi valoración sobre la universidad es positiva & & \\
\hline
\end{tabular}

Fuente: Elaboración propia.

Para el factor "Planificación docente" (tabla 4), el ítem que ha obtenido una puntuación media más baja es el relacionado con el desarrollo de asignaturas impartidas entre varios profesores $(\mathrm{M}=3,33 ; \mathrm{SD}=, 988)$. El alumnado considera que el reparto de los créditos entre varios docentes perjudica la docencia, pues debe existir una clara conexión entre ambos para la adecuada organización de la asignatura. No obstante, el alumnado del Máster de Secundaria de la Universidad Católica de Murcia sostiene cómo el sistema de evaluación utilizado por el profesorado contribuye al desarrollo de su aprendizaje $(\mathrm{M}=3,60 ; \mathrm{SD}=, 940)$. Igualmente, valora otros aspectos tales como: el planteamiento de diferentes tipos de actividades para favorecer la adquisición de competencias en el alumnado ( $\mathrm{M}=3,60 ; \mathrm{SD}=, 930)$; el desarrollo de un clima empático profesor-alumno $(\mathrm{M}=3,61 ; \mathrm{SD}=, 966) ;$ la adecuación de la metodología a las características del alumnado $(\mathrm{M}=3,64 ; \mathrm{SD}=, 928)$; la utilización adecuada de recursos materiales $(\mathrm{M}=3,64 ; \mathrm{SD}=, 973)$; el desarrollo de tutorías $(\mathrm{M}=3,64 ; \mathrm{SD}=, 960)$; la utilización de referencias bibliográficas actuales y de fácil acceso $(\mathrm{M}=3,79 ; \mathrm{SD}=, 934)$ así como de espacios virtuales $(\mathrm{M}=3,85$; $\mathrm{SD}=, 927)$. Se trata de aspectos vinculados al desarrollo de una metodología participativa, capaz de centrar el interés sobre la actividad del alumnado, teniendo en cuenta sus características e intereses individuales.

Para el factor "Convergencia europea en los planes de estudio" (tabla 5), los resultados obtenidos revelan cómo los estudiantes del Máster de Secundaria consideran que, gracias al proceso de Bolonia, los nuevos planes de estudio mejoran su formación en competencias $(\mathrm{M}=3,14 ; \mathrm{SD}=, 996)$ y contribuyen a la formación y desarrollo profesional del estudiante para su inserción socio-laboral $(\mathrm{M}=3,17 ; \mathrm{SD}=, 991)$, movilidad entre universidades europeas $(\mathrm{M}=3,18 ; \mathrm{SD}=1,058)$ y aprendizaje de una lengua extranjera $(\mathrm{M}=3,20 ; \mathrm{SD}=1,186)$. Se trata de cuestiones de vital importancia para lograr un aprendizaje competitivo que permita el desarrollo y la empleabilidad del alumnado a escala internacional.

No obstante, se muestran más convencidos acerca de las potencialidades de los nuevos planes de estudio para la homogeneización de los estudios universitarios a nivel europeo $(\mathrm{M}=3,21 ; \mathrm{SD}=, 983)$, la participación activa del alumnado en el proceso de aprendizaje $(\mathrm{M}=3,40 ; \quad \mathrm{SD}=, 892)$ y el trabajo autónomo del alumno $(\mathrm{M}=3,42 ; \quad \mathrm{SD}=1,059)$. 
Finalmente, se destaca que el alumnado considera necesaria la reducción de la ratio $(\mathrm{M}=3,44 ; \mathrm{SD}=1,128)$, a pesar de valorar adecuadamente las instalaciones ofrecidas por la universidad $(\mathrm{M}=3,77 ; \mathrm{SD}=1,070)$ y el Prácticum como complemento para su formación académica $(\mathrm{M}=4,02 ; \mathrm{SD}=, 979)$.

Tabla 4. Análisis descriptivo del factor "Planificación docente"

\begin{tabular}{lcc}
\hline \multicolumn{1}{c}{ ÍTEMS } & M & SD \\
\hline $\begin{array}{l}\text { Las asignaturas impartidas entre varios profesores facilitan el proceso de } \\
\text { enseñanza-aprendizaje }\end{array}$ & 3,33 &, 988 \\
$\begin{array}{l}\text { El sistema de evaluación utilizado ha contribuido al desarrollo de mi } \\
\text { aprendizaje }\end{array}$ & 3,60 &, 940 \\
$\begin{array}{l}\text { El profesorado plantea diferentes tipos de actividades para una mejor } \\
\text { adquisición de las competencias }\end{array}$ & 3,60 &, 930 \\
$\begin{array}{l}\text { La relación profesor-alumno permite crear un clima empático para } \\
\text { favorecer el proceso de enseñanza-aprendizaje }\end{array}$ & 3,61 &, 966 \\
$\begin{array}{l}\text { La metodología empleada por el profesor se adecúa a las características del } \\
\text { alumnado }\end{array}$ & 3,64 &, 928 \\
$\begin{array}{l}\text { Los recursos materiales de los que dispone el profesorado son suficientes y } \\
\text { adecuados }\end{array}$ & 3,64 &, 973 \\
$\begin{array}{l}\text { Las tutorías facilitan la comprensión y asimilación de contenidos propios } \\
\text { del título }\end{array}$ & 3,64 &, 960 \\
$\begin{array}{l}\text { En general, el profesorado utiliza referencias bibliográficas actuales y de } \\
\text { fácil acceso }\end{array}$ & 3,79 &, 934 \\
$\begin{array}{l}\text { El profesorado utiliza de manera adecuada los espacios virtuales para } \\
\text { difundir materiales y fomentar la enseñanza virtual }\end{array}$ & 3,85 &, 927 \\
\hline
\end{tabular}

Fuente: Elaboración propia.

Sin duda, se trata de aspectos generales vinculados al proceso de enseñanza-aprendizaje en Educación Superior, lo que exige tener en cuenta dicha valoración a partir de los referentes y creencias que posee el alumnado acerca del concepto "planificación docente". No obstante, supone un elemento de interés conocer la valoración del alumnado, pues es el principal agente educativo, destinatario directo del quehacer docente en el aula universitaria y al que se debe tener en consideración al proponer determinadas reformas que afectan al sistema universitario.

Tabla 5. Análisis descriptivo del factor "Convergencia europea en los planes de estudio"

\begin{tabular}{lcc}
\hline \multicolumn{1}{c}{ ÍTEMS } & M & SD \\
\hline $\begin{array}{l}\text { Los nuevos planes de estudios mejoran la formación en competencias del } \\
\text { alumnado universitario }\end{array}$ & 3,14 &, 996 \\
$\begin{array}{l}\text { Los nuevos planes de estudio contribuyen a la formación y al desarrollo } \\
\text { profesional de los estudiantes de cara a la inserción socio-laboral }\end{array}$ & 3,17 &, 991 \\
$\begin{array}{l}\text { La nueva normativa europea ha posibilitado la movilidad de los estudiantes } \\
\text { en las universidades }\end{array}$ & 3,18 & \multirow{2}{*}{1,058} \\
$\begin{array}{l}\text { La nueva normativa europea sobre educación universitaria ha contribuido } \\
\text { al aprendizaje de una lengua extranjera en el alumnado universitario }\end{array}$ & 3,20 & 1,186 \\
$\begin{array}{l}\text { La adaptación de los planes de estudio al contexto europeo ha favorecido la } \\
\text { homogeneización de los estudios universitarios a nivel europeo }\end{array}$ & 3,21 &, 983 \\
$\begin{array}{l}\text { La nueva normativa europea fomenta la participación activa del alumnado } \\
\text { en el proceso de aprendizaje }\end{array}$ & 3,40 &, 892 \\
$\begin{array}{l}\text { Los nuevos planes de estudio favorecen el trabajo autónomo del estudiante } \\
\text { Considero que debería reducirse el número de alumnos por grupo }\end{array}$ & 3,42 & 1,059 \\
$\begin{array}{l}\text { Las instalaciones de la universidad son adecuadas y se adaptan a mis } \\
\text { necesidades }\end{array}$ & 3,44 & 1,128 \\
El Prácticum complementa mi formación académica & 3,77 & 1,070 \\
\hline
\end{tabular}

Fuente: Elaboración propia. 
Respecto al factor "Organización docente" (tabla 6), los resultados revelan la inquietud de los estudiantes del Máster de Educación Secundaria ante el nuevo sistema de créditos $(\mathrm{M}=2,96 ; \mathrm{SD}=1,260)$, así como el número de convocatorias establecidas para los exámenes $(\mathrm{M}=3,12 ; \mathrm{SD}=1,152)$. No obstante, se muestran favorables ante la adecuación del calendario de exámenes a sus necesidades e intereses $(\mathrm{M}=3,48 ; \mathrm{SD}=1,072)$ y consideran que el calendario académico del título facilita su aprendizaje ( $\mathrm{M}=3,60$; $\mathrm{SD}=, 940$ ). De este modo, el actual sistema de créditos ECTS y las calificaciones a nivel europeo favorecen el reconocimiento de las cualificaciones profesionales y la movilidad internacional al tiempo que permite la colaboración entre universidades y el aprendizaje a lo largo de la vida para el alumnado.

Finalmente, se destaca cómo el alumnado de dicho título considera que su participación contribuye a la mejora del proceso de aprendizaje $(\mathrm{M}=3,73 ; \mathrm{SD}=, 923)$, manifestando existir coherencia entre los contenidos teóricos y las prácticas de las asignaturas $(\mathrm{M}=3,82 ; \mathrm{SD}=, 912)$ y realzando la figura del tutor de Prácticum como conexión entre el centro educativo y la universidad $(\mathrm{M}=4,04 ; \mathrm{SD}=1,015)$. Se apuesta, desde este punto de vista, por el desarrollo de la competencia de "aprender a aprender", frente a la acumulación memorística de información.

Centrados en la "Información académica" (tabla 7), los resultados obtenidos revelan cómo el alumnado del Máster de Secundaria no se muestra muy convencido acerca de la información recibida sobre los nuevos planes de estudio y sus implicaciones para sus estudios universitarios $(\mathrm{M}=2,84 ; \mathrm{SD}=1,160)$. De igual modo, afirman no haber recibido demasiada información sobre los nuevos planes de estudio a través de cualquier medio ajeno a la universidad $(\mathrm{M}=2,94 ; \mathrm{SD}=1,193)$. La estructura organizativa modificada en Educación Superior gracias al proceso de convergencia europea ha permitido la información para los agentes impulsores de tales cambios, dejando en un segundo plano la comunicación con el alumnado, siendo este el principal agente educativo del quehacer docente.

Tabla 6. Análisis descriptivo del factor "Organización docente"

\begin{tabular}{lcc}
\hline \multicolumn{1}{c}{ ÍTEMS } & M & SD \\
\hline $\begin{array}{l}\text { Conozco íntegramente el nuevo sistema de créditos } \\
\text { El número de convocatorias de los exámenes de las asignaturas del máster }\end{array}$ & 2,96 & 1,260 \\
$\begin{array}{l}\text { es suficiente } \\
\text { El calendario de exámenes se adecúa a mis intereses y necesidades }\end{array}$ & 1,152 \\
$\begin{array}{l}\text { académicas } \\
\text { El calendario académico del título facilita el aprendizaje del alumnado } \\
\text { universitario }\end{array}$ & 3,48 & 1,072 \\
$\begin{array}{l}\text { La participación del alumnado contribuye a la mejora del proceso de } \\
\text { aprendizaje }\end{array}$ &, 940 \\
$\begin{array}{l}\text { En general, existe coherencia entre los contenidos teóricos y las prácticas } \\
\text { de las asignaturas }\end{array}$ & 3,73 &, 923 \\
$\begin{array}{l}\text { Considero fundamental la figura del tutor de Prácticum para que haya una } \\
\text { conexión entre el centro educativo y la universidad }\end{array}$ & 4,04 & 1,012 \\
\hline
\end{tabular}

Fuente: Elaboración propia.

A pesar de todo, los estudiantes encuestados se muestran satisfechos con la información que disponen acerca de los aspectos académicos del título que cursan ( $\mathrm{M}=3,63$; $\mathrm{SD}=, 931)$, afirmando conocer las competencias propias del título $(\mathrm{M}=3,73 ; \mathrm{SD}=, 924)$ así como los resultados de aprendizaje $(\mathrm{M}=3,76 ; \mathrm{SD}=, 891)$. 
Tabla 7. Análisis descriptivo del factor "Información académica"

\begin{tabular}{lcc}
\hline \multicolumn{1}{c}{ ÍTEMS } & M & SD \\
\hline $\begin{array}{l}\text { Desde la universidad, se me ha informado acerca de los nuevos planes de } \\
\text { estudio y sus implicaciones para mis estudios }\end{array}$ & 2,84, & 1,160 \\
$\begin{array}{l}\text { He recibido informaciones acerca de los nuevos planes de estudio de la } \\
\text { Educación Superior por cualquier medio ajeno a la universidad }\end{array}$ & 2,94 & 1,193 \\
$\begin{array}{l}\text { Dispongo de suficiente información sobre los aspectos académicos del } \\
\text { título que estoy cursando }\end{array}$ & 3,63 & \multirow{2}{*}{, 931} \\
Conozco las competencias del título que estoy cursando & 3,73 &, 924 \\
Conozco los resultados de aprendizaje del título que estoy cursando & 3,76 &, 891 \\
\hline
\end{tabular}

Fuente: Elaboración propia.

Respecto a las posibilidades de especialización del título (tabla 8), el alumnado del Máster de Educación Secundaria considera que los nuevos planes de estudio permiten la especialización de su formación académica $(\mathrm{M}=3,48 ; \mathrm{SD}=, 977)$. Así pues, afirman conocer dichas posibilidades $(\mathrm{M}=4, \mathrm{OO} ; \mathrm{SD}=, 968)$ considerando cómo el Trabajo Fin de Máster contribuye a alcanzar las competencias propias del título $(\mathrm{M}=4,04 ; \mathrm{SD}=1,015)$.

Tabla 8. Análisis descriptivo del factor "Especialización del título"

\begin{tabular}{lcc}
\hline \multicolumn{1}{c}{ ÍTEMS } & M & SD \\
\hline $\begin{array}{l}\text { Considero que los nuevos planes de estudio permiten una mayor } \\
\text { especialización en mi titulación }\end{array}$ & 3,48 &, 977 \\
$\begin{array}{l}\text { Conozco las diferentes posibilidades de especialización establecidas por la } \\
\text { universidad en el título que estoy cursando }\end{array}$ & 4,00 &, 968 \\
$\begin{array}{l}\text { El Trabajo Fin de Grado/Máster me permite alcanzar las competencias del } \\
\text { título }\end{array}$ & 4,04 & \multirow{2}{*}{1,015} \\
\hline
\end{tabular}

Fuente: Elaboración propia.

\subsection{Diferencia de medias}

El análisis de varianza realizado (ANOVA) revela la existencia de diferencias estadísticamente significativas para algunos de los ítems del cuestionario (tabla 9). Por un lado, y teniendo en cuenta la edad del alumnado, las diferencias se localizan en las valoraciones de los estudiantes del Máster de Secundaria a la hora de considerar la contribución de la nueva normativa europea hacia el aprendizaje de una lengua extranjera $(\mathrm{F}(2,248)=3,186, \mathrm{p}=, 043)$. De igual modo, se aprecian diferencias en las percepciones del alumnado al analizar si estos han recibido informaciones acerca de los nuevos planes de estudio por cualquier medio ajeno a la propia institución universitaria $(\mathrm{F}(2,248)=3,932, \mathrm{p}=, 021)$. Así pues, se aprecian diferencias estadísticamente significativas en las percepciones del alumnado al considerar la calidad de la enseñanza recibida en el máster $(\mathrm{F}(2,247)=5,289, \mathrm{p}=, 006)$ así como el uso que el profesorado de dicho título hace de referencias bibliográficas actualizadas y de fácil acceso $(\mathrm{F}(2,247)=4,565, \mathrm{p}=, 011)$.

No obstante, la prueba de Tukey realizada a posteriori revela cómo dichas diferencias se sitúan entre el alumnado más joven que acaba de terminar sus estudios universitarios (cuyas edades oscilan entre los 20-25 años) y aquel otro de mayor edad (entre 26 y 30 años o más de 31 años) y ha accedido ya al mercado laboral o, por el contrario, desea complementar su formación académica. En este caso, y tal y como se muestra en la tabla adjunta, las valoraciones resultan ser más favorables para aquellos estudiantes del máster que poseen más edad, superando los 26 años. 
Tabla 9. ANOVA según la edad del alumnado del Máster de Secundaria

\begin{tabular}{|c|c|c|c|c|c|}
\hline ÍTEMS & $20 / 25$ & $\begin{array}{l}\text { M. } \\
26 / 30\end{array}$ & +31 & ANOVA & TUKEY \\
\hline $\begin{array}{l}\text { La nueva normativa europea sobre } \\
\text { educación universitaria ha contribuido al } \\
\text { aprendizaje de una lengua extranjera en el } \\
\text { alumnado universitario }\end{array}$ & $2.99^{*}$ & $3.41^{*}$ & 3.26 & $.043^{*}$ & $\begin{array}{l}20 / 25- \\
26 / 30\end{array}$ \\
\hline $\begin{array}{l}\text { He recibido informaciones acerca de los } \\
\text { nuevos planes de estudio por cualquier } \\
\text { medio ajeno a la universidad }\end{array}$ & $2.75^{*}$ & $3.22^{*}$ & 2.88 & $.021^{*}$ & $\begin{array}{l}20 / 25- \\
26 / 30\end{array}$ \\
\hline $\begin{array}{l}\text { La enseñanza recibida en el máster es de } \\
\text { calidad }\end{array}$ & $3.57 *$ & 3.61 & $\begin{array}{c}4.04 \\
*\end{array}$ & $.006^{*}$ & $20 / 25-+31$ \\
\hline $\begin{array}{l}\text { En general, el profesorado utiliza } \\
\text { referencias bibliográficas actuales y de } \\
\text { fácil acceso }\end{array}$ & $3.63^{*}$ & 3.80 & $\begin{array}{l}4.09 \\
*\end{array}$ & $.011^{*}$ & $20 / 25-+31$ \\
\hline
\end{tabular}

Fuente: Elaboración propia.

Por otro lado, y teniendo en consideración la especialidad por la que el alumnado cursa este Máster en Secundaria, el análisis de varianza (ANOVA) realizado revela diferencias estadísticamente significativas en sus percepciones (tabla 10). Así pues, dichas diferencias se sitúan en las valoraciones del alumnado a la hora de considerar la adecuación del calendario académico $(\mathrm{F}(4,244)=2,438, \mathrm{p}=, 048)$, la calidad de la enseñanza recibida $(\mathrm{F}(4,244)=3,057, \mathrm{p}=, 017)$ y la participación del alumnado para la contribución del proceso de enseñanza-aprendizaje $(\mathrm{F}(4,243)=2,920, \mathrm{p}=, 022)$. Finalmente, se destaca cómo las diferencias se sitúan a la hora de valorar el planteamiento, por parte del profesorado, de distintos tipos de actividades para mejorar la adquisición de competencias en el alumnado $(\mathrm{F}(2,245)=3,780, \mathrm{p}=$,005) así como la adecuación de los recursos materiales a utilizar en el aula $(\mathrm{F}(4,246)=4,393, \mathrm{p}=, 002)$.

Además, la prueba de Tukey realizada a posteriori revela cómo las percepciones del alumnado hacia el proceso de convergencia europea resultan más favorables para aquellos que cursan dicho título desde las especialidades de Filología e Idiomas y Humanidades, Ciencias Sociales y de la Empresa, frente a aquellos otros que lo hacen por las especialidades de Ciencia y Tecnología, Actividad Física y del Deporte y Artes.

Tabla 10. ANOVA según la especialidad del alumnado del Máster de Secundaria

\begin{tabular}{|c|c|c|c|c|c|c|c|}
\hline \multirow{2}{*}{ ÍTEMS } & \multicolumn{5}{|c|}{ M. } & \multirow{2}{*}{ ANOVA } & \multirow{2}{*}{ TUKEY } \\
\hline & CT & HUM & FI & AFD & $\mathbf{A}$ & & \\
\hline $\begin{array}{l}\text { El calendario académico del título } \\
\text { facilita el aprendizaje del alumnado } \\
\text { universitario }\end{array}$ & $3.38 *$ & 3.68 & $3.91^{*}$ & 3.53 & 3.40 & $.048 *$ & CT-FI \\
\hline $\begin{array}{l}\text { La enseñanza recibida en el máster } \\
\text { es de calidad }\end{array}$ & $3.70^{*}$ & 3.74 & $3.98^{*}$ & 3.47 & 2.80 & $.017 *$ & CT-FI \\
\hline $\begin{array}{l}\text { La participación del alumnado } \\
\text { contribuye a la mejora del proceso } \\
\text { de aprendizaje }\end{array}$ & 3.78 & 3.82 & $3.93^{*}$ & $3.43^{*}$ & $3.00 *$ & $.022^{*}$ & $\begin{array}{l}\text { AFD- } \\
\text { FI; } \\
\text { A-FI }\end{array}$ \\
\hline $\begin{array}{l}\text { El profesorado plantea diferentes } \\
\text { tipos de actividades para una mejor } \\
\text { adquisición de las competencias }\end{array}$ & 3.51 & $3.67 *$ & 3.93 & $3.41 *$ & 2.60 & $.005^{*}$ & $\begin{array}{c}\text { HUM- } \\
\text { AFD }\end{array}$ \\
\hline $\begin{array}{l}\text { Los recursos materiales de que } \\
\text { dispone el profesorado son } \\
\text { suficientes y apropiados }\end{array}$ & 3.49 & $3.83 *$ & 3.98 & $3.31^{*}$ & 3.20 & $.002^{*}$ & $\begin{array}{c}\text { HUM- } \\
\text { AFD }\end{array}$ \\
\hline
\end{tabular}

CT: Ciencia y Tecnología; HUM: Humanidades, Ciencias Sociales y de la Empresa; FI=Filología e Idiomas; AFD: Actividad Física y del Deporte; A: Artes.

Fuente: Elaboración propia. 


\section{Discusión y conclusiones}

Esta investigación permite conocer las percepciones del alumnado del Máster en Formación del Profesorado acerca del proceso de convergencia europea. La evaluación que estos agentes educativos hacen del proceso formativo supone un elemento de vital importancia para la calidad y mejora de la Educación Superior, entendida esta como un proceso de búsqueda hacia la excelencia, la perfección y/o la adecuación a un propósito pre-establecido que promueve la transformación del sistema universitario.

Así pues, y tras el análisis de los resultados, se destaca la indiferencia del alumnado al considerar la mejora de la educación universitaria gracias a la nueva reestructuración de las titulaciones en grados y másteres. Del mismo modo, Coterón, Franco y Gil (2012) aluden a la valoración negativa del alumnado hacia los cambios introducidos en el sistema universitario español gracias al proceso de convergencia europea. Además, se insiste en el desconocimiento del alumnado ante el nuevo sistema de créditos ECTS. Estos datos guardan relación con los aportados en distintas investigaciones (FontMayolas y Masferrer, 2010; Leo y Cubo, 2012; Méndez, 2008), las cuales revelan la escasa información de que dispone el alumnado acerca de la instauración del proceso de convergencia europea en Educación Superior.

No obstante, la valoración del alumnado sobre la actuación docente es un indicador de calidad (Lukas et al., 2014). De este modo, el alumnado encuestado valora de forma positiva la universidad así como el plan de estudios de su titulación; a su vez, realza la importancia del Prácticum así como del tutor de Prácticum como complemento para su formación académica. Estos resultados contradicen los presentados por Hernández y Carrasco (2012), para quienes el alumnado del Máster de Secundaria no se muestra satisfecho con el tiempo establecido para el título que están cursando, la vinculación entre la teoría y la práctica en el desarrollo de programas formativos así como con la coordinación entre el profesorado.

Los encuestados sostienen cómo los nuevos títulos mejoran la formación en competencias del alumnado, amplían las posibilidades de inserción socio-laboral, movilidad de los estudiantes, etc. Sin embargo, subrayan la falta de información sobre los nuevos planes de estudio, a pesar de conocer la descripción de las competencias y resultados de aprendizaje expuestos en las guías docentes del título que están cursando y estar de acuerdo con la adecuación de las posibilidades de especialización que les ofrece dicho título de posgrado.

El alumnado encuestado refleja su malestar hacia el desarrollo de asignaturas impartidas por varios profesores, pues consideran que esto no facilita su aprendizaje. De distinto modo, considera adecuado el desarrollo del proceso de enseñanza y aprendizaje puesto en marcha desde dicha institución: sistema de evaluación, actividades, relación profesoralumno, metodología, recursos utilizados, etc. Dichas percepciones entran en contradicción con las valoraciones de expertos en formación de profesores de Educación Secundaria descritas por Benarroch (2011). Estos critican la ausencia de criterios de temporalización entre módulos así como la falta de coherencia entre los modelos de enseñanza utilizados y lo que dichos profesionales deben aplicar en el aula.

Además, dicha investigación concluye cómo es el alumnado de mayor edad quien muestra unas percepciones más favorables hacia el proceso de convergencia europea. El hecho de haber realizado estudios universitarios anteriormente sujetos a otros sistemas 
educativos y planes de estudio tradicionales permite al alumnado establecer comparaciones más objetivas basadas en el análisis de la realidad y la experiencia formativa.

Igualmente, cabe preguntarse el nivel de exigencia establecido por el alumnado al cursar dichos estudios de posgrado, lo cual puede influir en sus valoraciones acerca del actual proceso de convergencia europea. Ello implica un mayor peso para el trabajo autónomo del alumnado, la diversificación de actividades de aprendizaje, el planteamiento didáctico de la tutoría, el uso de las TIC, etc. (Tejedor y García-Valcárcel, 2007). El alumnado de mayor edad aboga por el establecimiento de un sistema de Educación Superior basado en el trabajo activo del alumnado, capaz de manipular el conocimiento, actualizarlo, seleccionar lo que es apropiado para cada situación, aprender de forma permanente, entender lo que se aprende y extrapolarlo a nuevos contextos (Esteve, 2003). No obstante, y en contraposición a los resultados obtenidos, el alumno más joven se muestra reacio a los cambios en el sistema universitario español y prefiere limitarse a ser receptor y reproductor de conocimientos trasmitidos por el profesor (Gargallo, Suárez y Ferreras, 2007; Pozo y Pérez, 2009).

En cuanto a la especialidad por la que cursar dicho Máster, los resultados demuestran cómo el alumnado de las áreas de Filología e Idiomas y Humanidades, Ciencias Sociales y de la Empresa muestra unas percepciones más favorables hacia el proceso de convergencia europea que el alumnado que lo hace por la especialidad de Ciencia y Tecnología, Actividad Física y del Deporte y Artes. Ello puede estar debido al hecho de que el alumnado propio de la especialidad de Humanidades ha desarrollado estudiantes universitarios anteriores vinculados al ámbito de la educación, por lo que es capaz de analizar dicho cambio en el sistema universitario español con un mayor conocimiento que aquel otro cuyos estudios universitarios iniciales estuvieron referidos al ámbito de las Ciencias, el Deporte o las Artes.

Sin duda, un estudio de enorme interés que permite analizar las percepciones hacia el proceso de convergencia europea en estudiantes del Máster de Educación Secundaria. Así pues, y a través del análisis de tales valoraciones, el sistema universitario podrá establecer mapas de indicadores que reflejen las principales demandas e inquietudes del alumnado para así dar respuesta a las mismas; de este modo, sus valoraciones deben servir como indicador de la calidad de la gestión y desarrollo de la Educación Superior (Álvarez, Chaparro y Reyes, 2015). No obstante, la escasa muestra con la que se ha llevado a cabo esta investigación no nos permite garantizar la generalización de los resultados a otras muestras. Al mismo tiempo, el uso exclusivo del cuestionario como instrumento de recogida de datos puede generar problemas de deseabilidad social y sinceridad al cumplimentarlo.

Carrasco (2012) destaca cómo entre las cuestiones más valoradas por el alumnado se encuentran la evaluación continua, la relación personalizada con el docente, la posibilidad de estudiar o trabajar en otros países europeos, la disminución de la ratio, etc. Por ello, y de cara a futuras investigaciones, se plantea explorar dichos indicadores a fondo, valorando las percepciones del alumnado hacia estos aspectos y su implicación para el proceso de enseñanza-aprendizaje en Educación Superior. Además, puede resultar interesante conocer las valoraciones del profesorado que imparte docencia en dicho título para, de este modo, contrastar la información arrojada y establecer sugerencias de mejora. Asimismo, se pretende comparar dichas percepciones en 
alumnado de Posgrado con aquellos otros que se encuentran realizando estudios de grado para poder comparar tales valoraciones.

\section{Referencias}

Alcaraz, N., Fernández, M. y Sola, M. (2012). La voz del alumnado en los procesos de evaluación docente universitaria. Revista Iberoamericana de Evaluación Educativa, 5(2), 26-39.

Álvarez, J., Chaparro, E. M. y Reyes, D. E. (2015). Estudio de la satisfacción de los estudiantes con los servicios educativos brindados por instituciones de Educación Superior del Valle de Toluca. REICE. Revista Iberoamericana sobre Calidad, Eficacia y Cambio en Educación, $13(2), 5-26$.

Benarroch, A. (2011). Diseño y desarrollo del Máster en Profesorado de Educación Secundaria durante su primer año de implantación. Revista Eureka sobre Enseñanza y Divulgación de las Ciencias, 8(1), 20-40.

Benso, M. C. y Pereira, M. C. (Eds.). (2003). El profesorado de enseñanza secundaria. Retos ante el nuevo milenio. Ourense: Concello de Ourense/Fundación Santa María/Universidad de Vigo.

Carrasco, C. (2012). ¿Cómo se está aplicando el Plan Bolonia? Una visión crítica de los alumnos de segundo curso del Grado de Psicología. En L. del Río e I. Teva (Comps.), IX Foro Internacional sobre la Evaluación de la Calidad de la Investigación y de la Educación Superior (pp. 532-537). Granada: Asociación Española de Psicología Conductual.

Castaño, E., Benito, A., Portela, A. y Rodríguez, R. M. (2007). Repercusiones en los alumnos de primer curso de la implantación del Espacio Europeo. Revista Complutense de Educación, 18(1), 199-216.

Cachón, J., López, I., Romero, S., Zagalaz, M. L. y González, C. (2015). Opinión de docentes y estudiantes del máster de secundaria sobre las aportaciones de este a la formación del profesorado, la calidad docente y los intereses personales. Magister, 27(1), 1-10. doi:10.1016/j.magis.2015.03.001

Cid, A., Sarmiento, J. A. y Pérez, A. (2012). Las concepciones sobre la enseñanza de los estudiantes que realizaron el CAP y el Máster de Educación Secundaria en la Universidad de Vigo. Revista de Investigación en Educación, 10(1), 100-114.

Coterón, J., Franco, E., y Gil, J. (2012). Opinión del alumnado sobre la implantación del Espacio Europeo de Educación Superior en estudios de Ciencias de la Actividad Física y del Deporte. Revista Complutense de Educación, 23(1), 191-206. doi:10.5209/rev_rced.2012.v23.n1.39109

De Pablos, J., Colás, P., González, T. y Jiménez, R. (2007). La adaptación de las universidades al Espacio Europeo de Educación Superior. Un procedimiento metodológico para el diseño de planes estratégicos. Revista de Investigación Educativa, 25(2), 533-554.

Esteve, J. M. (2003). La Tercera Revolución Educativa. La educación en la sociedad del conocimiento. Barcelona: Paidós.

Fernández-Molina, M., González, V. y Del Molino, G. (2011). Perfil del alumnado universitario de Educación Infantil. Un estudio descriptivo desde los inicios del Espacio Europeo de Educación Superior hasta los Estudios de Grado. Revista de Investigación Educativa, 29(1), 187-203. 
Font-Mayolas, S. y Masferrer, L. (2010). Conocimientos y actitudes de estudiantes universitarios respecto al Espacio Europeo de Educación Superior. Revista de Formación e Innovación Educativa Universitaria, 3(2), 88-96.

Gargallo, B., Suárez, J. y Ferreras, A. (2007): Estrategias de aprendizaje y rendimiento académico en estudiantes universitarios. Revista de Investigación Educativa, 2, 42 1-441.

González, J. C., Jiménez, J. R. y Pérez, H. M. (2011). El nuevo modelo formativo del profesorado de Educación Secundaria y su proceso de implantación en las universidades andaluzas. Revista Fuentes, 11, 67-85.

González, M. T. (2008). Educación para la ciudadanía: Implicaciones para el centro escolar como organización. En Sociedad Española de Pedagogía (Eds.), Educación ciudadanía y convivencia. Diversidad y sentido social de la educación. Actas del XIV Congreso Nacional y III Iberoamericano de Pedagogía (pp. 277-298). Zaragoza: Autor.

González-Sanmamed, M. (2009). Una nueva oportunidad para la formación inicial del profesorado de Educación Secundaria. Revista de Educación, 350, 57-78.

Gutiérrez-Herrera, J. A., Guadalupe, M. A., Santamaría, D., Cifuentes, G., Ramírez, M. I., Schnarch, D., Acevedo, S., Gómez, L. A., Fernanda, M. A., Rodríguez, E. F. y Díaz, J. D. (2012). La evaluación como camino en la cultura pedagógica y el mejoramiento de la labor docente. Revista Iberoamericana de Evaluación Educativa, 5(2), 265-283.

Hargreaves, A. y Shirley, D. (2012). La cuarta vía. El prometedor futuro del cambio educativo. Barcelona: Octaedro.

Hernández, M. J. y Carrasco, V. (2012). Percepciones de los estudiantes del Máster en Formación del Profesorado de Educación Secundaria: Fortalezas y debilidades del nuevo modelo formativo. Enseñanza \& Teaching, 30(2), 127-152.

Imbernón, F. (2007). La formación inicial del profesorado de secundaria, ¿sigue siendo un tema pendiente? Aula de Innovación Educativa, 161, 5-6.

Leo, M. y Cubo, S. (2012). El Espacio Europeo de Educación Superior. Actitudes del alumnado de la Universidad de Extremadura. Campo Abierto, 31(1), 29-50.

López, M. C., Pérez-García, M. P. y Rodríguez, M. J. (2015). Concepciones del profesorado universitario sobre la formación en el marco del espacio europeo de educación superior. Revista de Investigación Educativa, 33(1), 179-194.

Lukas, J. F., Santiago, K., Etxeberria, J. y Lizasoain, L. (2014). Adaptación al Espacio Europeo de Educación Superior de un cuestionario de opinión del alumnado sobre la docencia de su profesorado. RELIEVE. Revista Electrónica de Investigación y Evaluación Educativa, 20(1), 120. doi:10.7203/relieve.20.1.3812

Manso-Ayuso, J. y Martín, E. (2014). Valoración del Máster de Formación de Profesorado de Secundaria: Estudio de casos en dos universidades. Revista de Educación, 364, 145-169.

Medina, A. (2010). Problemas emergentes en la formación inicial del profesorado de secundaria. En I. González (Coord.), El nuevo profesor de secundaria. La formación docente en el marco del Espacio Europeo de Educación Superior (pp. 135-150). Barcelona: Graó.

Méndez, R. M. (2008). Los jóvenes universitarios y su (des)orientación ante los nuevos retos que plantea el EEES. Educatio Siglo XXI, 26, 197-224.

Olmedo, E. M. (2013). Enfoques de aprendizaje de los estudiantes y metodología docente: Evolución hacia el nuevo sistema de formación e interacción propuesta en el EEES. Revista de Investigación Educativa, 31(2), 411-429. doi:10.6018/rie.31.2.133501 
Otero, M. C., Ferro, C. y Vila, M. (2012). Satisfacción del alumnado ante la implantación del modelo del EEES. Análisis comparativo. Hekademos: Revista educativa digital, 12, 35-42.

Perales, M. J., Jornet, J. M. y González-Such, J. (2014). Tendencias en las políticas de formación y evaluación del profesorado en la Educación Superior en España. Revista Iberoamericana de Evaluación Educativa, 7(2), 53-54.

Pérez, E., y Rodríguez, J. (2012). Reflexiones del alumnado del Máster de Secundaria ante el Espacio Europeo de Educación Superior. Revista Riaices, o(1), 31-50.

Pozo, J. I. y Pérez, M. P. (2009). Psicología del aprendizaje universitario: La formación en competencias. Madrid: Morata.

Puelles, M. (Coord.). (2009). Profesión y vocación docente: Presente y futuro. Madrid: Biblioteca Nueva.

Reoyo, N., Carbonero, M. A., Freitas, A. y Valdivieso, J. A. (2012). La percepción de los futuros profesores sobre los docentes de Educación Secundaria. International Journal of Developmental and Educational Psychology INFAD Revista de Psicología, 1(2), 389-396.

Rué, J., Mateo, P. y Muñoz, A. (2012). Informe sobre la evaluación de la calidad de la docencia en las universidades españolas. Barcelona: Universitat Autónoma de Barcelona / Red Estatal de Docencia Universitaria (RED-U).

Sans de Castro, R. (2013). Experiencia y valoración del máster de secundaria. Iber. Didáctica de las Ciencias Sociales, Geografía e Historia, 73, 65-72.

Serrano, R. y Pontes, A. (2015). Expectativas ante la formación inicial entre el alumnado del Máster de Profesorado en Enseñanza Secundaria. Revista de Investigación Educativa, 33(2), 489-505. doi:10.6018/rie.33.2.203471

Tejedor, F. J. y García-Valcárcel, A. (2007). Causas del bajo rendimiento del estudiante universitario (en opinión de los profesores y alumnos). Propuestas de mejora en el marco del EEES. Revista de Educación, 342, 443-474.

Timostsuk, I. y Ugaste, A. (2010). Student teachers' professional identity. Teaching and Teacher Education, 26(8), 1563-1570. doi:10.1016/j.tate.2010.06.008

Vilches, A. y Gil-Pérez, D. (2010). Máster de formación inicial del profesorado de Enseñanza Secundaria. Algunos análisis y propuestas. Revista Eureka sobre Enseñanza y Divulgación de las Ciencias, 7(3), 661-666.

\section{Breve CV de la autora}

\section{$\mathrm{M}^{\mathrm{a}}$ del Carmen Pegalajar Palomino}

Doctora por la Universidad de Jaén. Licenciada en Psicopedagogía y Diplomada en Maestro, especialidad en Educación Primaria. Máster en Educación Especial por la Universidad de Huelva. Profesora del Departamento de Pedagogía de la Universidad de Jaén. Sus líneas de investigación están centradas en la atención a la diversidad y la formación del docente. E-mail:mcpegala@ujaen.es 